\title{
Distributed Robust Attitude Tracking of Multiple Spacecraft with Disturbances and Unmodelled Dynamics
}

\author{
Dapeng Yang and Xiangdong Liu \\ State Key Laboratory for Intelligent Control \& Decision of Complex Systems, Beijing Institute of Technology, Beijing 100081, China \\ Correspondence should be addressed to Dapeng Yang; yangdp1988@163.com
}

Received 26 August 2014; Accepted 24 September 2014

Academic Editor: Housheng Su

Copyright (C) 2015 D. Yang and X. Liu. This is an open access article distributed under the Creative Commons Attribution License, which permits unrestricted use, distribution, and reproduction in any medium, provided the original work is properly cited.

\begin{abstract}
This paper considers the distributed robust attitude tracking problem of multiple spacecraft subject to disturbances and unmodelled dynamics. We designed a distributed robust attitude controller for each spacecraft using the relative attitudes and relative angular velocities of neighbors to ensure that the attitude tracking errors between the leader and the followers converge to zero under the condition that the communication graph among the followers is undirected and connected and at least one follower has the access to the leader. The control algorithm achieves robust attitude tracking under the existence of the disturbances and unmodelled dynamics by selecting the control gains according to the given condition.
\end{abstract}

\section{Introduction}

In the recent ten years, many researchers of the control field began to study the coordinated control of multiagent systems because of its broad applications including formation, flocking, and cooperative control [1-4]. It is wellknown that cooperative attitude control of multiple rigid bodies is an important research direction and has received much attention in the last decade. The existing literature of multiagent systems contains the work with integrator dynamics and general linear dynamics $[1,2,4-6]$. The topic of our paper, cooperative attitude tracking for multiple spacecraft, is more difficult. References [7-9] have considered the attitude control problem of single spacecraft or single rigid body by using some methods such as adaptive control and sliding mode control. For the attitude tracking problem of multiple rigid bodies or multiple spacecraft, distributed control strategies seem to be a proper method because there are some advantages such as less communication and decentralized computing [10].

Many researchers in the field of control engineering have considered the distributed attitude control problem of multiple rigid bodies. A leader-follower strategy for attitude coordination was studied in [11], while the cooperative attitude control problem was considered in [12]. The authors in [13] adopted the contraction analysis theory to guarantee the exponential convergence of attitude synchronization problem of multiple spacecraft. The communication topology in [13] is a bidirectional ring. Using quaternion to describe the spacecraft's attitude, [14] studied the distributed attitude synchronization problem. In contrast, the authors in [15] used modified Rodriguez parameters (MRPs) to represent the attitude of the spacecraft. Under the assumption that the communication topology is a directed spanning tree, [16] addressed the distributed attitude synchronization problem for multiple spacecraft with unknown inertia matrices. In a word, all the existing works above mainly considered the cases where the exact model knowledge of each spacecraft is known or the cases where the external disturbance does not exist. However, there might exist some disturbances and unmodelled dynamics in practical applications. References [17-19] considered the distributed coordinated control problem for multimanipulator systems or decentralized consensus problem for multiagent systems. The semiglobal leader-following consensus problem of linear multiagent systems with input saturation via low-gain feedback was investigated in $[20,21]$ based on state feedback and output feedback.

In this paper, we consider the distributed robust attitude tracking problem subject to disturbances and unmodelled dynamics for multiple spacecraft. In this paper, we adopt MRPs to describe the attitude for the spacecraft. We design 
a robust controller for each spacecraft in a distributed manner using the relative attitudes and the angular velocities of neighbors. Under the assumption that the communication topology among the followers is undirected and at least one follower can get the information from the leader, the proposed controller could guarantee that the combined errors between the leader spacecraft and the follower spacecraft would converge to zero. Although $[22,23]$ dealt with the robust consensus tracking problem for multiagent system where the agent dynamics were restricted to being single or double integrators with disturbances and unmodelled dynamics. In contrast to $[22,23]$ which only considered the robust tracking problem with linear dynamics, this paper considers the attitude tracking problem of multiple spacecraft where the attitude dynamics of spacecraft are totally nonlinear. The proposed controller solves the robust attitude tracking problem in the presence of disturbances and unmodelled dynamics by selecting the control gains according to the given condition. Under the proposed control strategy, only a subgroup of the followers have the access to the leader; that is, the robust attitude tracking can be achieved using only the neighboring information.

The structure of this paper is arranged as follows. Some existing results and mathematical preliminaries are introduced in Section 2. Section 3 investigated the distributed robust attitude tracking controllers. Section 4 represents the simulation examples. The conclusion of this paper is Section 5 .

\section{Mathematical Preliminaries}

2.1. Notation and Graph Theory. $I_{p}$ means the identity matrix of dimension $p . \mathbf{R}^{n \times n}$ represent the set of $n \times n$ real matrices. Let $T$ represent the transpose for real matrices. Let $A \otimes B$ be the Kronecker product of matrices $A$ and $B$. Let $\|x\|$ be the 2 -norm of a vector $x \cdot \operatorname{diag}\left(A_{1}, \ldots, A_{N}\right)$ denotes a block-diagonal matrix with matrices $A_{i}$, on its diagonal, $i=$ $1, \ldots, N$. We say a vector $x \in \mathscr{L}_{\infty}$, when all the elements of $x$ are bounded.

A directed graph $\mathscr{G}$ is a pair $(\mathscr{V}, \mathscr{E})$, where $\mathscr{V}=$ $\left\{v_{1}, \ldots, v_{N}\right\}$ is a set of nodes and $\mathscr{E} \subseteq \mathscr{V} \times \mathscr{V}$ is a set of edges, in which an edge is represented by an ordered pair of distinct nodes. For an edge $\left(v_{i}, v_{j}\right)$, we call node $v_{i}$ the parent node, node $v_{j}$ the child node, and $v_{i}$ a neighbor of $v_{j}$. If $(i, j) \in \mathscr{E}$ means $(j, i) \in \mathscr{E}$, we say the graph is undirected. A path from node $v_{i_{1}}$ to node $v_{i_{l}}$ is a sequence of ordered edges of the form $\left(v_{i_{k}}, v_{i_{k+1}}\right), k=1, \ldots, l-1$. The adjacency matrix $A=\left[a_{i j}\right] \in \mathbf{R}^{N \times N}$ associated with the directed graph $\mathscr{G}$ is defined by $a_{i i}=0, a_{i j}=1$ if $\left(v_{j}, v_{i}\right) \in \mathscr{E}$ and $a_{i j}=0$ otherwise. The Laplacian matrix $L=\left[l_{i j}\right] \in \mathbf{R}^{N \times N}$ is defined as $l_{i i}=\sum_{j=0, j \neq i}^{N} a_{i j}$ and $l_{i j}=-a_{i j}, i \neq j$.

Lemma 1 (see [24]). Zero is a simple eigenvalue of $L$ and the associated eigenvector is $\mathbf{1}$ if and only if the undirected graph is connected, where $\mathbf{1}=[1, \ldots, 1]^{T} \in \mathbf{R}^{N}$ is a unitary column vector. All of the nonzero eigenvalues of $L$ are real and positive for an undirected graph.
2.2. Attitude Dynamics. In this paper, we consider the distributed robust attitude tracking problem for a group of $N+$ 1 spacecrafts. We adopt MRPs to represent the attitude of a spacecraft with respect to the inertial frame. The MRP vector $\sigma_{i} \in \mathbf{R}^{3}$ is defined by $\sigma_{i}=\widehat{\mathbf{e}}_{i} \tan \left(\phi_{i} / 4\right)$ for the $i$ th spacecraft, where $\widehat{\mathbf{e}}_{i}$ is the Euler axis and $\phi_{i}$ is the Euler angle [25]. In our following discussion, it is assumed that the leader spacecraft is indexed by 0 and the follower spacecrafts are labeled by $1, \ldots, N$. For the $i$ th follower spacecraft, the attitude dynamics is described by [26]

$$
\begin{gathered}
J_{i} \dot{\omega}_{i}=-\omega_{i}^{\times} J_{i} \omega_{i}+u_{i}+f_{i}^{\prime}, \\
\dot{\sigma}_{i}=G\left(\sigma_{i}\right) \omega_{i}, \quad i=1, \ldots, N,
\end{gathered}
$$

where $J_{i} \in \mathbf{R}^{3 \times 3}$ is the inertia matrix, $\omega_{i} \in \mathbf{R}^{3}$ denotes the attitude angular velocity with respect to the inertial frame in the body-fixed frame, $u_{i} \in \mathbf{R}^{3}$ is the control input, $\omega_{i}^{\times} \in \mathbf{R}^{3 \times 3}$ is the skew-symmetric matrix such that $\omega_{i}^{\times} v=\omega_{i} \times v$ for any vector $v \in \mathbf{R}^{3}, f_{i}^{\prime}$ represents the disturbances and unmodelled dynamics, and

$$
G\left(\sigma_{i}\right)=\frac{1}{2}\left(\frac{1-\sigma_{i}^{T} \sigma_{i}}{2} I_{3}+\sigma_{i}^{\times}+\sigma_{i} \sigma_{i}^{T}\right) .
$$

Rewrite the dynamics equation to get a simpler form as follows:

$$
\begin{aligned}
& \dot{\omega}_{i}=-J_{i}^{-1} \omega_{i}^{\times} J_{i} \omega_{i}+J_{i}^{-1} u_{i}+f_{i}, \\
& \dot{\sigma}_{i}=G_{i} \omega_{i},
\end{aligned}
$$

where $f_{i}=J_{i}^{-1} f_{i}^{\prime}$. Note that we define a new form $f_{i}(t)$ instead of $J_{i}^{-1} f_{i}^{\prime}(t)$ to represent the disturbances and unmodelled dynamics, which does not affect the overall analysis in this paper. This modification is only for simplicity in the subsequent discussion. We assume that the leader is not a real spacecraft, but just a reference signal. So we do not consider the disturbances and unmodelled dynamics for the leader.

We assume that there is no access to any follower spacecraft from the leader. And the leader's information can only be obtained by a subset of the followers. We use an undirected graph to represent the communication topology of the $N$ followers. The access of the followers to the leader is represented by a diagonal matrix $A_{0}=\operatorname{diag}\left(a_{10}, \ldots, a_{N 0}\right) \in$ $\mathbf{R}^{N \times N} \cdot a_{i 0}=1, i=1, \ldots, N$, if the $i$ th spacecraft has the access to the leader and $a_{i 0}=0$ otherwise. To facilitate the stability analysis of the closed-loop control system, we make the following assumptions on $f_{i}(t)$ and the communication graph among the $N+1$ spacecraft. Our control objective is to drive the follower spacecraft's attitudes $\sigma_{i}, i=1, \ldots, N$, to follow the leader spacecraft's attitude $\sigma_{0}$ in the presence of the disturbances and unmodelled dynamics existing in the follower spacecraft's attitude dynamics. It is reasonable that there exist external disturbances and parameter uncertainties in the attitude dynamics for the followers in some applications.

Assumption 2. The disturbances term $f_{i}(t)$ and the first-order and second-order time derivatives of $f_{i}(t)$ are bounded; that is, $f_{i}(t), \dot{f}_{i}(t), \ddot{f}_{i}(t) \in \mathscr{L}_{\infty}, i=1, \ldots, N$. 
Assumption 3. The graph $\mathscr{G}$ is connected and at least one follower has the access to the leader.

\section{Main Results}

This section considers the distributed robust attitude tracking problem for multiple spacecraft described by (3). The followers' attitudes are desired to follow the leader in the presence of the disturbances and unmodelled dynamics. Note that the attitude of the leader is a time-varying signal. The distributed attitude tracking problem is defined as follows.

Definition 4. The distributed robust attitude tracking problem is said to be solved, if the local control laws $u_{i}, i=$ $1, \ldots, N$, are designed for (3) in the presence of disturbances and unmodelled dynamics such that $\lim _{t \rightarrow \infty}\left\|\sigma_{i}-\sigma_{0}\right\|=0$ and $\lim _{t \rightarrow \infty}\left\|\omega_{i}-\omega_{0}\right\|=0, i=1, \ldots, N$.

Using the local information available to the $i$ th spacecraft, the angular velocity tracking error $e_{\omega_{i}}$, the attitude tracking error $e_{\sigma_{i}}$, and the combined tracking error $e_{i}$ for each follower spacecraft at each time are defined by

$$
\begin{gathered}
e_{\omega_{i}}=\sum_{j=1}^{N} a_{i j}\left(\omega_{i}-\omega_{j}\right)+a_{i 0}\left(\omega_{i}-\omega_{0}\right), \\
e_{\sigma_{i}}=\sum_{j=1}^{N} a_{i j}\left(\sigma_{i}-\sigma_{j}\right)+a_{i 0}\left(\sigma_{i}-\sigma_{0}\right), \\
e_{i}=e_{\omega_{i}}+\alpha e_{\sigma_{i}}, \quad i, j=1, \ldots, N
\end{gathered}
$$

where $a_{i j}$ is the $(i, j)$ th element of the adjacency matrix and $\alpha \in \mathbf{R}$ is a positive constant.

A distributed robust attitude tracking controller is proposed for each follower as

$$
\begin{aligned}
& u_{i}=J_{i}\left(-\widehat{f}_{i}+\rho_{i}+a_{i 0} \dot{\omega}_{0}-k_{c} e_{i}-\alpha G_{i} \omega_{i}\right), \\
& \dot{\hat{f}}_{i}=k_{1} \dot{e}_{i}+k_{2} \operatorname{sgn}\left(e_{i}\right)+k_{3} e_{i}, \quad i=1, \ldots, N,
\end{aligned}
$$

using only the local information of neighboring spacecraft, where $\rho_{i}=J_{i}^{-1} \omega_{i}^{\times} J_{i} \omega_{i}, k_{c}, k_{1}, k_{2}$, and $k_{3} \in \mathbf{R}$ are positive constant control gains to be determined and $\operatorname{sgn}(\cdot)$ is the sign function. Since the disturbances and unmodelled dynamics term $f_{i}$ is unknown, we use its estimate $\widehat{f}_{i}$ to obtain the control law $u_{i}$. We can see that $\hat{f}_{i}$ is calculated by the combined tracking error $e_{i}$ for each spacecraft. To facilitate the further analysis, a group of concatenated vectors and diagonal matrices are defined as

$$
\begin{aligned}
\omega=\left[\begin{array}{lll}
\omega_{1}^{T} & \cdots & \omega_{N}^{T}
\end{array}\right]^{T}, & \sigma=\left[\begin{array}{lll}
\sigma_{1}^{T} & \cdots & \sigma_{N}^{T}
\end{array}\right]^{T}, \\
u=\left[\begin{array}{lll}
u_{1}^{T} & \cdots & u_{N}^{T}
\end{array}\right]^{T}, & f=\left[\begin{array}{lll}
f_{1}^{T} & \cdots & f_{N}^{T}
\end{array}\right]^{T}, \\
\widehat{f}=\left[\begin{array}{lll}
\hat{f}_{1}^{T} & \cdots & \hat{f}_{N}^{T}
\end{array}\right]^{T}, & e_{\omega}=\left[\begin{array}{lll}
e_{\omega_{1}}^{T} & \cdots & e_{\omega_{N}}^{T}
\end{array}\right]^{T},
\end{aligned}
$$

$$
\begin{aligned}
e_{\sigma} & =\left[\begin{array}{lll}
e_{\sigma_{1}}^{T} & \cdots & e_{\sigma_{N}}^{T}
\end{array}\right]^{T}, \quad e=\left[\begin{array}{lll}
e_{1}^{T} & \cdots & e_{N}^{T}
\end{array}\right]^{T}, \\
\rho & =\left[\begin{array}{lll}
\rho_{1}^{T} & \cdots & \rho_{N}^{T}
\end{array}\right]^{T}, \quad G=\operatorname{diag}\left(G_{1}, \ldots, G_{N}\right), \\
J & =\operatorname{diag}\left(J_{1}, \ldots, J_{N}\right) .
\end{aligned}
$$

Then, it can be derived that

$$
\begin{aligned}
e_{\omega} & =\left(M \otimes I_{3}\right) \omega-A_{0} \otimes \omega_{0}, \\
e_{\sigma} & =\left(M \otimes I_{3}\right) \sigma-A_{0} \otimes \sigma_{0}, \\
e & =e_{\omega}+\alpha e_{\sigma} \\
& =\left(M \otimes I_{3}\right)(\omega+\alpha \sigma)-A_{0} \otimes\left(\omega_{0}+\alpha \sigma_{0}\right),
\end{aligned}
$$

where $M \triangleq L+A_{0}$. The time derivative of the concatenated combined tracking error $e$ is given by

$$
\begin{aligned}
\dot{e}= & \dot{e}_{\omega}+\alpha \dot{e}_{\sigma} \\
= & \left(M \otimes I_{3}\right)\left(-\rho+J^{-1} u+f+\alpha G \omega\right) \\
& -A_{0} \otimes\left(\dot{\omega}_{0}+\alpha \dot{\sigma}_{0}\right) \\
= & \left(M \otimes I_{3}\right)\left(-\rho+J^{-1} J\left(-\widehat{f}+\rho+A_{0} \otimes \dot{\omega}_{0}\right.\right. \\
& \left.\left.\quad-k_{c} e-\alpha G \omega\right)+f+\alpha G \omega\right) \\
= & \left(M \otimes I_{0} \otimes\left(\dot{\omega}_{0}+\alpha \dot{\sigma}_{0}\right)\left(f-\widehat{f}-k_{c} e+A_{0} \otimes \dot{\omega}_{0}\right)\right. \\
& -A_{0} \otimes\left(\dot{\omega}_{0}+\alpha \dot{\sigma}_{0}\right) .
\end{aligned}
$$

In addition, define a filtered tracking error $r(t) \in \mathbf{R}^{3 \times N}$ by

$$
k_{c}\left(M \otimes I_{3}\right) r=k_{c} \dot{e}+e,
$$

deriving that $\dot{e}=\left(M \otimes I_{3}\right) r-\left(1 / k_{c}\right) e$ and

$$
\begin{aligned}
r & =\left(M \otimes I_{3}\right)^{-1} \dot{e}+\frac{1}{k_{c}}\left(M \otimes I_{3}\right)^{-1} e \\
& =\int_{0}^{t} m d \tau-\widehat{f}-k_{c} e+\frac{1}{k_{c}}\left(M \otimes I_{3}\right)^{-1} e,
\end{aligned}
$$

where $\int_{0}^{t} m d \tau=f+A_{0} \otimes \dot{\omega}_{0}-\left(M \otimes I_{3}\right)^{-1} A_{0} \otimes\left(\dot{\omega}_{0}+\alpha \dot{\sigma}_{0}\right)$. Using $\dot{e}=\left(M \otimes I_{3}\right) r-\left(1 / k_{c}\right) e$, the time derivative of $r$ is given by

$$
\begin{aligned}
\dot{r}= & m-\dot{\hat{f}}-k_{c} \dot{e}+\frac{1}{k_{c}}\left(M \otimes I_{3}\right)^{-1} \dot{e} \\
= & m-k_{2} \operatorname{sgn}(e)-k_{3} e-k_{1}\left(M \otimes I_{3}\right) r+\frac{k_{1}}{k_{c}} e \\
& -k_{c}\left(M \otimes I_{3}\right) r+e+\frac{1}{k_{c}} r-\frac{1}{k_{c}^{2}}\left(M \otimes I_{3}\right)^{-1} e .
\end{aligned}
$$


Remark 5. It can be concluded that the convergence of the filtered tracking error $r(t)$ guarantees the convergence of the concatenated combined tracking error $e(t)$, which can be proved using the Lyapunov-based analysis by selecting a Lyapunov function $V(e)=(1 / 2) e^{T} e$.

Lemma 6 (see [22]). A function $s(t) \in \mathbf{R}$ defined as follows is positive semidefinite:

$$
\begin{aligned}
s(t)= & e(0)^{T} k_{2} \operatorname{sgn}(e(0))-e(0)^{T} m(0) \\
& -\int_{0}^{t} r^{T}\left(M \otimes I_{3}\right)\left(m-k_{2} \operatorname{sgn}(e)\right) d \tau,
\end{aligned}
$$

if the control gains $k_{2}$ and $k_{c}$ satisfy

$$
k_{2}>\|m(t)\|+k_{c}\|\dot{m}(t)\| .
$$

Theorem 7. Suppose that Assumptions 2 and 3 hold. The distributed robust attitude tracking problem of the spacecraft in (3) is solved by the distributed attitude tracking control law (5) if the control gains are selected such that

$$
\begin{gathered}
k_{2}>\|m(t)\|+k_{c}\|\dot{m}(t)\|, \\
k_{3}=\frac{k_{1}}{k_{c}}+1, \\
M \otimes I_{3}-\frac{1}{k_{c}^{2}} I_{3 \times N}>0 .
\end{gathered}
$$

Proof. Note that $M=L+A_{0}$ and $L$ is the Laplacian matrix associated with graph $\mathscr{G}$. Under the Assumption 2, $M$ is positive definite [27]. Then, it can be seen that

$$
\begin{aligned}
e= & \left(M \otimes I_{3}\right)(\omega+\alpha \sigma)-A_{0} \otimes\left(\omega_{0}+\alpha \sigma_{0}\right) \\
= & \left(M \otimes I_{3}\right)(\omega+\alpha \sigma)-A_{0} \otimes\left(\omega_{0}+\alpha \sigma_{0}\right) \\
& -L \otimes\left(\omega_{0}+\alpha \sigma_{0}\right) \\
= & \left(M \otimes I_{3}\right)\left[(\omega+\alpha \sigma)-I_{N} \otimes\left(\omega_{0}+\alpha \sigma_{0}\right)\right],
\end{aligned}
$$

where we have used the fact that $L \otimes\left(\omega_{0}+\alpha \sigma_{0}\right)=\left(L \otimes I_{3}\right)\left(I_{N} \otimes\right.$ $\left.\left(\omega_{0}+\alpha \omega_{0}\right)\right)=0$ because 1 is the eigenvector of the Laplacian matrix $L$ associated with the simple eigenvalue 0 according to Lemma 1 and $I_{N} \otimes\left(\omega_{0}+\alpha \omega_{0}\right) \in \operatorname{span}\{\mathbf{1}\}$. Then, it follows from (15) and the positive definiteness of the matrix $M$ that $e=0$ if and only if

$$
\omega_{0}+\alpha \sigma_{0}=\cdots=\omega_{N}+\alpha \sigma_{N}
$$

which, as proved in [28], in turn implies that

$$
\lim _{t \rightarrow \infty}\left\|\sigma_{i}-\sigma_{0}\right\|=0, \quad \lim _{t \rightarrow \infty}\left\|\omega_{i}-\omega_{0}\right\|=0, \quad i=1, \ldots, N .
$$

So, the distributed robust attitude tracking problem is solved if and only if $e(t)=0$ as $t \rightarrow \infty$.

Choose the Lyapunov function candidate

$$
V(t)=\frac{1}{2} e^{T} e+\frac{1}{2} r^{T}\left(M \otimes I_{3}\right) r+s,
$$

where $s$ is defined in (12). From Assumption 2, $f_{i}(t)$, $\dot{f}_{i}(t), \ddot{f}_{i}(t) \in L_{\infty}, i=1, \ldots, N$, which implies that $\dot{F}$ and $\ddot{F}$ are both bounded. In view of the boundedness of the initial state of the leader spacecraft, we know that $\dot{\omega}_{0}(0), \ddot{\omega}_{0}(0), \dot{\sigma}_{0}(0), \ddot{\sigma}_{0}(0) \in L_{\infty}$. Based on the definition of $m(t)$ in (10), we can see that $\|m(t)\|$ and $\|\dot{m}(t)\|$ are both bounded. Then, it follows from Lemma 6, the positive definiteness of the matrix $M$, and the first condition in (14) that the Lyapunov function candidate $V(t)$ is positive definite. Then the time derivative of $V(t)$ along the trajectory of (10), (11), and (12) is given by

$$
\begin{aligned}
\dot{V}(t)= & e^{T} \dot{e}+r^{T}\left(M \otimes I_{3}\right) \dot{r}+\dot{s} \\
= & e^{T}\left(\left(M \otimes I_{3}\right) r-\frac{1}{k_{c}} e\right)+r^{T}\left(M \otimes I_{3}\right) \\
& \times\left(m-k_{2} \operatorname{sgn}(e)-k_{3} e-k_{1}\left(M \otimes I_{3}\right) r+\frac{k_{1}}{k_{c}} e\right. \\
& \left.\quad k_{c}\left(M \otimes I_{3}\right) r+e+\frac{1}{k_{c}} r-\frac{1}{k_{c}^{2}}\left(M \otimes I_{3}\right)^{-1} e\right) \\
& -r^{T}\left(M \otimes I_{3}\right)\left(m-k_{2} \operatorname{sgn}(e)\right) .
\end{aligned}
$$

By using the second condition in (14), we can obtain that

$$
\begin{aligned}
\dot{V}(t)= & -\frac{1}{k_{c}} e^{T} e-k_{1} r^{T}\left(M \otimes I_{3}\right)\left(M \otimes I_{3}\right) r \\
& -k_{c} r^{T}\left(M \otimes I_{3}\right)\left(\left(M \otimes I_{3}\right)-\frac{1}{k_{c}^{2}} I_{3 \times N}\right) r \\
& +r^{T}\left(\left(M \otimes I_{3}\right)-\frac{1}{k_{c}^{2}} I_{3 \times N}\right) e .
\end{aligned}
$$

Since

$$
\begin{aligned}
r^{T}( & \left.\left(M \otimes I_{3}\right)-\frac{1}{k_{c}^{2}} I_{3 \times N}\right) e \\
= & -\frac{1}{2 k_{c}}\left[e-k_{c}\left(\left(M \otimes I_{3}\right)-\frac{1}{k_{c}^{2}} I_{3 \times N}\right) r\right]^{T} \\
& \times\left[e-k_{c}\left(\left(M \otimes I_{3}\right)-\frac{1}{k_{c}^{2}} I_{3 \times N}\right) r\right] \\
& +\frac{1}{2 k_{c}} k_{c}^{2} r^{T}\left(\left(M \otimes I_{3}\right)-\frac{1}{k_{c}^{2}} I_{3 \times N}\right)^{2} r+\frac{1}{2 k_{c}} e^{T} e
\end{aligned}
$$

it follows that

$$
\begin{aligned}
\dot{V}(t)= & -\frac{1}{k_{c}} e^{T} e-k_{1} r^{T}\left(M \otimes I_{3}\right)\left(M \otimes I_{3}\right) r \\
& -k_{c} r^{T}\left(M \otimes I_{3}\right)\left(\left(M \otimes I_{3}\right)-\frac{1}{k_{c}^{2}} I_{3 \times N}\right) r
\end{aligned}
$$




$$
\begin{aligned}
& -\frac{1}{2 k_{c}}\left[e-k_{c}\left(\left(M \otimes I_{3}\right)-\frac{1}{k_{c}^{2}} I_{3 \times N}\right) r\right]^{T} \\
& \times\left[e-k_{c}\left(\left(M \otimes I_{3}\right)-\frac{1}{k_{c}^{2}} I_{3 \times N}\right) r\right] \\
& +\frac{1}{2 k_{c}} k_{c}^{2} r^{T}\left(\left(M \otimes I_{3}\right)-\frac{1}{k_{c}^{2}} I_{3 \times N}\right)^{2} r+\frac{1}{2 k_{c}} e^{T} e \\
& =-\frac{1}{2 k_{c}} e^{T} e-k_{1} r^{T}\left(M \otimes I_{3}\right)\left(M \otimes I_{3}\right) r \\
& -\frac{1}{2 k_{c}}\left[e-k_{c}\left(\left(M \otimes I_{3}\right)-\frac{1}{k_{c}^{2}} I_{3 \times N}\right) r\right]^{T} \\
& \times\left[e-k_{c}\left(\left(M \otimes I_{3}\right)-\frac{1}{k_{c}^{2}} I_{3 \times N}\right) r\right] \\
& -k_{c} r^{T}\left(M \otimes I_{3}\right)\left(\left(M \otimes I_{3}\right)-\frac{1}{k_{c}^{2}} I_{3 \times N}\right) r \\
& +\frac{1}{2} k_{c} r^{T}\left(\left(M \otimes I_{3}\right)-\frac{1}{k_{c}^{2}} I_{3 \times N}\right)^{2} r \\
& =-\frac{1}{2 k_{c}} e^{T} e-k_{1} r^{T}\left(M \otimes I_{3}\right)\left(M \otimes I_{3}\right) r \\
& -\frac{1}{2 k_{c}}\left[e-k_{c}\left(\left(M \otimes I_{3}\right)-\frac{1}{k_{c}^{2}} I_{3 \times N}\right) r\right]^{T} \\
& \times\left[e-k_{c}\left(\left(M \otimes I_{3}\right)-\frac{1}{k_{c}^{2}} I_{3 \times N}\right) r\right] \\
& -k_{c} r^{T}\left[\left(M \otimes I_{3}\right)^{2}-\frac{1}{k_{c}^{2}}\left(M \otimes I_{3}\right)-\frac{1}{2}\left(M \otimes I_{3}\right)^{2}\right. \\
& \left.-\frac{1}{2 k_{c}^{4}} I_{3 \times N}+\frac{1}{k_{c}^{2}}\left(M \otimes I_{3}\right)\right] r \\
& =-\frac{1}{2 k_{c}} e^{T} e-k_{1} r^{T}\left(M \otimes I_{3}\right)\left(M \otimes I_{3}\right) r \\
& -\frac{1}{2 k_{c}}\left[e-k_{c}\left(\left(M \otimes I_{3}\right)-\frac{1}{k_{c}^{2}} I_{3 \times N}\right) r\right]^{T} \\
& \times\left[e-k_{c}\left(\left(M \otimes I_{3}\right)-\frac{1}{k_{c}^{2}} I_{3 \times N}\right) r\right] \\
& -\frac{1}{2} k_{c} r^{T}\left[\left(M \otimes I_{3}\right)+\frac{1}{k_{c}^{2}} I_{3 \times N}\right] \\
& \times\left[\left(M \otimes I_{3}\right)-\frac{1}{k_{c}^{2}} I_{3 \times N}\right] r \text {, }
\end{aligned}
$$

where we have used the equation $M^{T}=M$ since the graph $\mathscr{G}$ is undirected.

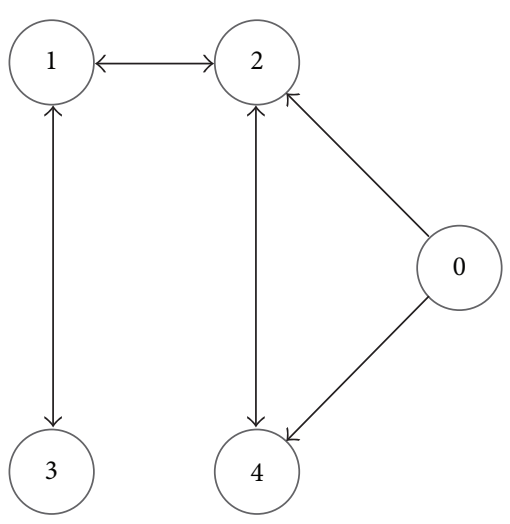

FIGURE 1: The communication topology of spacecraft.

In view of the third condition in (14), we obtain that $M \otimes I_{3}-\left(1 / k_{c}^{2}\right) I_{3 \times N}>0$. Then, if follows from (22) that $\dot{V}(t) \leq 0$. Since $V(t)>0$ and $\dot{V}(t) \leq 0$, it is easy to see that $V(t) \leq V(0) \in L_{\infty}$. From the definition of $V(t)$, we can see that $e(t), r(t) \in L_{\infty}$. Based on the boundedness of $f(t), e(t)$, $\dot{\omega}_{0}(t), \dot{\sigma}_{0}(t),(5)$, and (8), we can obtain $\widehat{f}(t), \dot{e}(t) \in L_{\infty}$. Then, it follows from (10) and the boundedness of $m(t), \dot{\hat{f}}(t)$, and $\dot{e}(t)$ that $\dot{r}(t) \in L_{\infty}$, which in turn guarantees that $\ddot{V}(t)=$ $f(e, \dot{e}, r, \dot{r})$ is bounded. According to Barbalat's lemma [29], we get that $\dot{V}(t) \rightarrow 0$ as $t \rightarrow \infty$; that is, $\lim _{t \rightarrow \infty} e(t)=0$, $i=1, \ldots, N$. Thus, the distributed robust attitude tracking problem is solved.

Remark 8. The cooperative attitude synchronization problem for multiple rigid bodies has been solved in $[15,30]$. However, the authors in $[15,30]$ only considered the nominal model of the attitude dynamics, while in this paper the disturbances and unmodelled dynamics are contained during the analysis. This paper proposed a sufficient condition for achieving distributed robust attitude tracking between the following spacecraft and the leader, which is a more challenging task because of the disturbances and unmodelled dynamics.

Remark 9. Compared to $[22,31]$ where the robust consensus tracking problems were considered and the agent dynamics were restricted to being single or double integrator, this paper considers robust attitude tracking of multiple spacecraft where the attitude dynamics of spacecraft are nonlinear. Additionally, [32, 33] considered the rendezvous problem and the adaptive consensus problem for multiple mobile linear agents with preserved network connectivity, which is an interesting topic for the distributed cooperative control of multiple spacecraft. However, it is more challenging and cannot be obtained easily by extending the results in our paper. We will consider the network connectivity preserving case in our future research.

\section{Simulation Examples}

Consider a group of five spacecraft whose communication topology is given by Figure 1. The inertia matrices of the 
TABLE 1: Spacecraft inertias.

\begin{tabular}{|c|c|}
\hline Number & Parameters $/\left(\mathrm{kg} \cdot \mathrm{m}^{2}\right)$ \\
\hline$J_{0}$ & {$\left[\begin{array}{lllllll}1.1 & 0.35 & 0.45 ; 0.35 & 1.0 & 0.5 ; 0.45 & 0.5 & 1.3\end{array}\right]$} \\
\hline$J_{1}$ & {$\left[\begin{array}{lllllll}1 & 0.1 & 0.1 ; 0.1 & 0.1 & 0.1 ; 0.1 & 0.1 & 0.9\end{array}\right]$} \\
\hline$J_{2}$ & {$\left[\begin{array}{lllllll}1.5 & 0.2 & 0.3 ; 0.2 & 0.9 & 0.4 ; 0.3 & 0.4 & 2.0\end{array}\right]$} \\
\hline$J_{3}$ & {$\left[\begin{array}{llllllll}0.8 & 0.1 & 0.2 ; 0.1 & 0.7 & 0.3 ; 0.2 & 0.3 & 1.1\end{array}\right]$} \\
\hline$J_{4}$ & {$\left[\begin{array}{lllllll}1.2 & 0.3 & 0.7 ; 0.3 & 0.9 & 0.2 ; 0.7 & 0.2 & 1.4\end{array}\right]$} \\
\hline
\end{tabular}

spacecraft are shown in Table 1 . We choose the initial attitude $\sigma_{i}(0), \omega_{i}(0), i=0, \ldots, 4$, randomly. In this section, we assume that the leader spacecraft's information can only be obtained by spacecrafts 1 and 2 . We design the control input $u_{0}$ as

$$
u_{0}=J_{0}\left[\rho_{0}-k_{0}\left(\omega_{0}-\omega_{0}^{d}\right)\right] \text {, }
$$

where $\rho_{0}=J_{0}^{-1} \omega_{0}^{\times} J_{0} \omega_{0}$ and $k_{0}=\operatorname{diag}(1,1,1)$, to track a given angular velocity $\omega_{0}^{d}=[0.2 \sin (0.3 t) ; 0.1 \sin (0.4 t)$; $0.15 \cos (0.5 t)]$.

To verify the theoretical result of our paper, let the constants in Theorem 7 be $k_{c}=10, k_{1}=0.1, k_{2}=0.003$, $k_{3}=1.01$, and $\alpha=1$. The disturbances and unmodelled dynamics $f_{i}^{\prime}(t), i=1, \ldots, 4$, for the follower spacecraft are given by

$$
\begin{aligned}
f_{i}^{\prime}(t) & =\left[\begin{array}{l}
f_{i 1}^{\prime}(t) \\
f_{i 2}^{\prime}(t) \\
f_{i 3}^{\prime}(t)
\end{array}\right] \\
= & {\left[\begin{array}{c}
\left(0.03-\frac{i}{100}\right) \sin (0.3 t) \\
\left(0.04-\frac{i}{100}\right) \cos (0.3 t) \\
\left(0.03-\frac{i}{100}\right) \sin \left(0.3 t+\frac{\pi}{4}\right)
\end{array}\right] . }
\end{aligned}
$$

The attitudes and the attitude angular velocities of the five spacecrafts are shown in Figures 2 and 3, from which we can see that the distributed robust attitude tracking problem is solved provided that the disturbances and unmodelled dynamics exist in the attitude dynamics. The tracking errors $e_{i}, i=1, \ldots, 4$, of the follower spacecraft are given in Figure 4, which clearly converge to zero as $t \rightarrow \infty$.

\section{Conclusion}

In this paper, we considered the distributed robust attitude tracking problem of multiple spacecraft with disturbances and unmodelled dynamics. We designed distributed robust controllers for multiple spacecraft based on the relative attitudes and relative attitude angular velocities of neighbors to track the leader's time-varying attitude in the presence of disturbances and unmodelled dynamics. The proposed control algorithm achieves robust attitude tracking for the case that the communication graph among follower spacecrafts is an undirected connected graph and at least one follower has the access to the leader by selecting the control
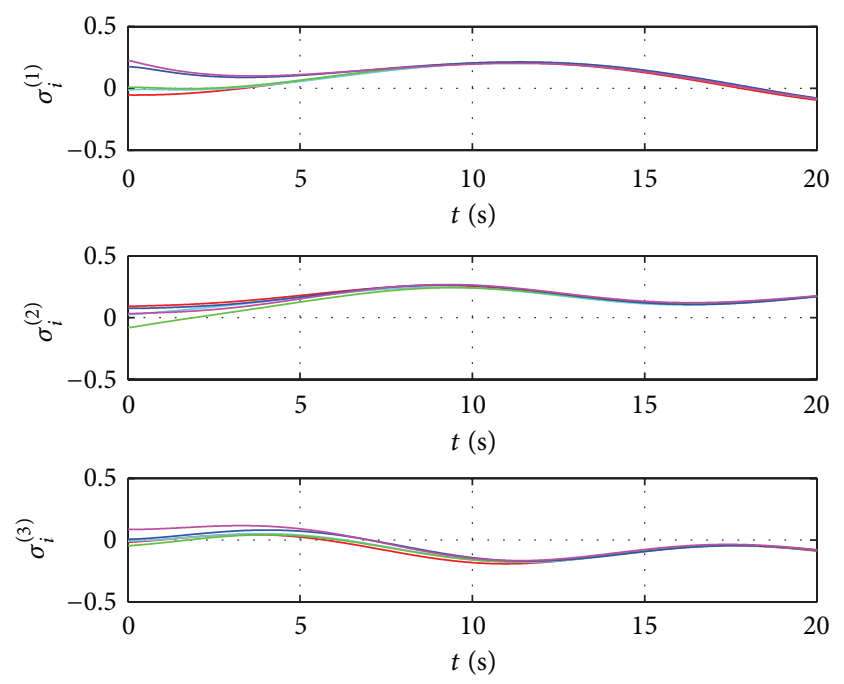

FIGURE 2: The attitudes under the robust controller (5).
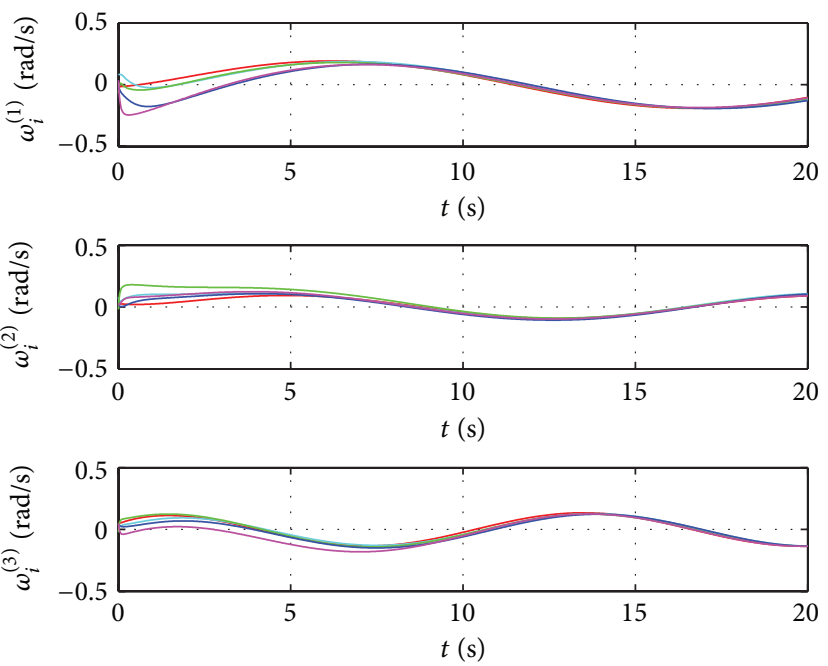

FIGURE 3: The angular velocities under the robust controller (5).
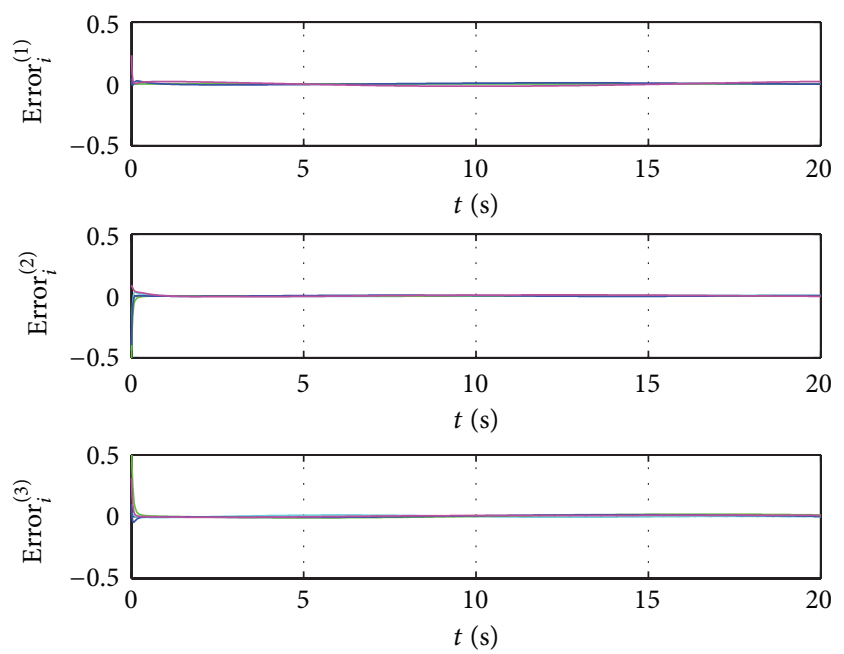

FIGURE 4: The tracking errors under the robust controller (5). 
gains according to the given condition. The distributed robust attitude tracking of multiple spacecraft without velocity measurements and robust attitude tracking under directed communication topologies are interesting topics for future research.

\section{Conflict of Interests}

The authors declare that there is no conflict of interests regarding the publication of this paper.

\section{References}

[1] R. Olfati-Saber and R. M. Murray, "Consensus problems in networks of agents with switching topology and time-delays," IEEE Transactions on Automatic Control, vol. 49, no. 9, pp. 15201533, 2004.

[2] W. Ren and R. W. Beard, "Consensus seeking in multiagent systems under dynamically changing interaction topologies," IEEE Transactions on Automatic Control, vol. 50, no. 5, pp. 655661, 2005

[3] W. Ren, R. W. Beard, and E. M. Atkins, "Information consensus in multivehicle cooperative control," IEEE Control Systems Magazine, vol. 27, no. 2, pp. 71-82, 2007.

[4] Z. Li, Z. Duan, G. Chen, and L. Huang, "Consensus of multiagent systems and synchronization of complex networks: a unified viewpoint," IEEE Transactions on Circuits and Systems I: Regular Papers, vol. 57, no. 1, pp. 213-224, 2010.

[5] Y. Hong, G. Chen, and L. Bushnell, "Distributed observers design for leader-following control of multi-agent networks," Automatica, vol. 44, no. 3, pp. 846-850, 2008.

[6] Z. Li, Z. Duan, and G. Chen, "On $H_{\infty}$ and $\mathrm{H}_{2}$ performance regions of multi-agent systems," Automatica, vol. 47, no. 4, pp. 797-803, 2011.

[7] G. Q. Xing and S. A. Parvez, "Nonlinear attitude state tracking control for spacecraft," Journal of Guidance, Control, and Dynamics, vol. 24, no. 3, pp. 624-626, 2001.

[8] W. Luo, Y.-C. Chu, and K.-V. Ling, "Inverse optimal adaptive control for attitude tracking of spacecraft," IEEE Transactions on Automatic Control, vol. 50, no. 11, pp. 1639-1654, 2005.

[9] H. Wong, M. S. De Queiroz, and V. Kapila, "Adaptive tracking control using synthesized velocity from attitude measurements," Automatica, vol. 37, no. 6, pp. 947-953, 2001.

[10] W. Ren and R. Beard, Distributed Consensus in Multi-Vehicle Cooperative Control: Theory and Applications, Springer, 2008.

[11] P. K. C. Wang, F. Y. Hadaegh, and K. Lau, "Synchronized formation rotation and attitude control of multiple free-flying spacecraft," Journal of Guidance, Control, and Dynamics, vol. 22, no. 1, pp. 28-35, 1999.

[12] D. V. Dimarogonas, P. Tsiotras, and K. J. Kyriakopoulos, "Leader-follower cooperative attitude control of multiple rigid bodies," Systems and Control Letters, vol. 58, no. 6, pp. 429-435, 2009.

[13] S.-J. Chung, U. Ahsun, and J.-J. E. Slotine, "Application of synchronization to formation flying spacecraft: lagrangian approach," Journal of Guidance, Control, and Dynamics, vol. 32, no. 2, pp. 512-526, 2009.

[14] A. Abdessameud and A. Tayebi, "Attitude synchronization of a group of spacecraft without velocity measurements," IEEE Transactions on Automatic Control, vol. 54, no. 11, pp. 26422648, 2009.
[15] W. Ren, "Distributed cooperative attitude synchronization and tracking for multiple rigid bodies," IEEE Transactions on Control Systems Technology, vol. 18, no. 2, pp. 383-392, 2010.

[16] Z. Li and Z. Duan, "Distributed adaptive attitude synchronization of multiple spacecraft," Science China Technological Sciences, vol. 54, no. 8, pp. 1992-1998, 2011.

[17] Z. Hou, L. Cheng, M. Tan, and X. Wang, "Distributed adaptive coordinated control of multi-manipulator systems using neural networks," in Robot Intelligence: An Advanced Knowledge Processing Approach, pp. 49-69, Springer, London, UK, 2010.

[18] L. Cheng, Z.-G. Hou, M. Tan, Y. Lin, and W. Zhang, "Neuralnetwork-based adaptive leader-following control for multiagent systems with uncertainties," IEEE Transactions on Neural Networks, vol. 21, no. 8, pp. 1351-1358, 2010.

[19] Z.-G. Hou, L. Cheng, and M. Tan, "Decentralized robust adaptive control for the multiagent system consensus problem using neural networks," IEEE Transactions on Systems, Man, and Cybernetics Part B: Cybernetics, vol. 39, no. 3, pp. 636-647, 2009.

[20] H. Su, M. Z. Q. Chen, J. Lam, and Z. Lin, "Semi-global leaderfollowing consensus of linear multi-agent systems with input saturation via low gain feedback," IEEE Transactions on Circuits and Systems I: Regular Papers, vol. 60, no. 7, pp. 1881-1889, 2013.

[21] H. Su, M. Z. Q. Chen, X. Wang, and J. Lam, "Semiglobal observer-based leader-following consensus with input saturation," IEEE Transactions on Industrial Electronics, vol. 61, no. 6, pp. 2842-2850, 2014.

[22] G. Hu, "Robust consensus tracking for an integrator-type multiagent system with disturbances and unmodelled dynamics," International Journal of Control, vol. 84, no. 1, pp. 1-8, 2011.

[23] G. Hu, "Robust consensus tracking of a class of second-order multi-agent dynamic systems," Systems and Control Letters, vol. 61, no. 1, pp. 134-142, 2012.

[24] R. Olfati-Saber, J. A. Fax, and R. M. Murray, "Consensus and cooperation in networked multi-agent systems," Proceedings of the IEEE, vol. 95, no. 1, pp. 215-233, 2007.

[25] M. D. Shuster, "Survey of attitude representations," The Journal of the Astronautical Sciences, vol. 41, no. 4, pp. 439-517, 1993.

[26] P. Tsiotras, "Further passivity results for the attitude control problem," IEEE Transactions on Automatic Control, vol. 43, no. 11, pp. 1597-1600, 1998.

[27] Y. Hong, J. Hu, and L. Gao, "Tracking control for multiagent consensus with an active leader and variable topology," Automatica, vol. 42, no. 7, pp. 1177-1182, 2006.

[28] Z.-X. Li and B.-L. Wang, "Robust attitude tracking control of spacecraft in the presence of disturbances," Journal of Guidance, Control, and Dynamics, vol. 30, no. 4, pp. 1156-1159, 2007.

[29] J. Slotine and W. Li, Applied Nonlinear Control, Prentice Hall, 1991.

[30] Z. Meng, W. Ren, and Z. You, "Decentralized cooperative attitude tracking using modified Rodriguez parameters," in Proceedings of the 48th IEEE Conference on Decision and Control held jointly with 28th Chinese Control Conference (CDC/CCC '09), pp. 853-858, December 2009.

[31] S. Khoo, L. Xie, and Z. Man, "Robust finite-time consensus tracking algorithm for multirobot systems," IEEE/ASME Transactions on Mechatronics, vol. 14, no. 2, pp. 219-228, 2009.

[32] H. Su, X. Wang, and G. Chen, "Rendezvous of multiple mobile agents with preserved network connectivity," Systems \& Control Letters, vol. 59, no. 5, pp. 313-322, 2010.

[33] H. Su, G. Chen, X. Wang, and Z. Lin, "Adaptive secondorder consensus of networked mobile agents with nonlinear dynamics," Automatica, vol. 47, no. 2, pp. 368-375, 2011. 


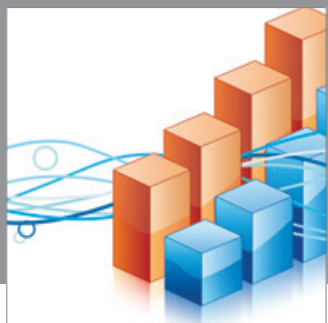

Advances in

Operations Research

mansans

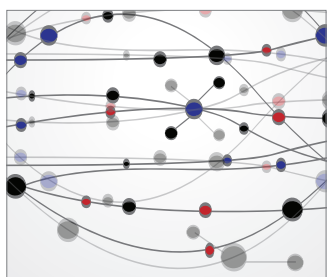

The Scientific World Journal
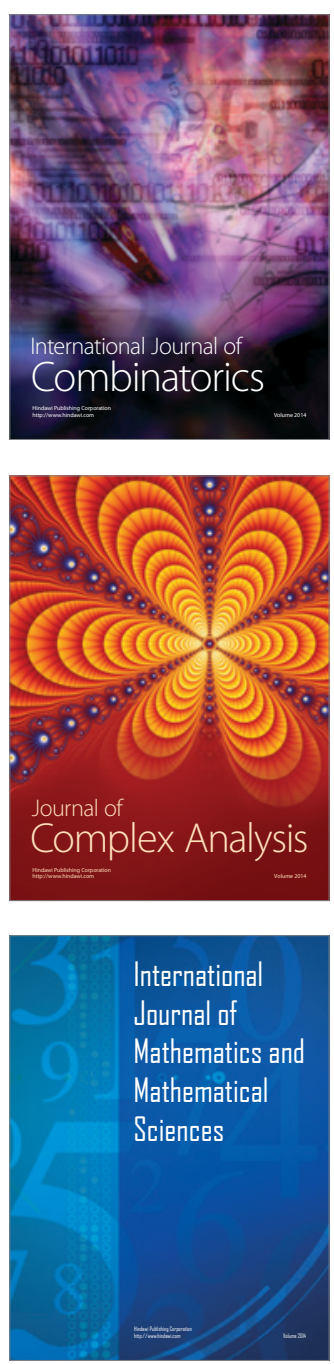
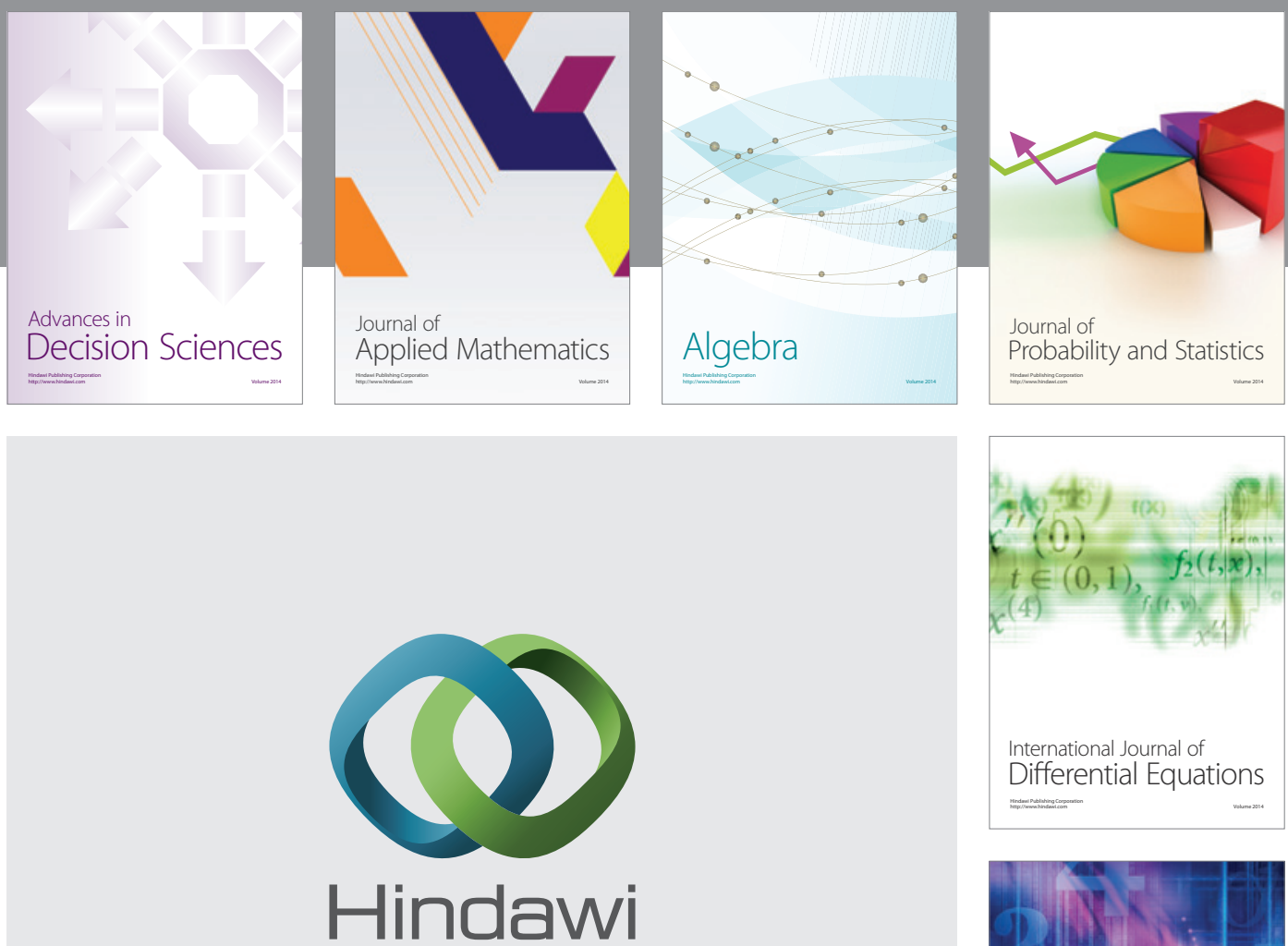

Submit your manuscripts at http://www.hindawi.com
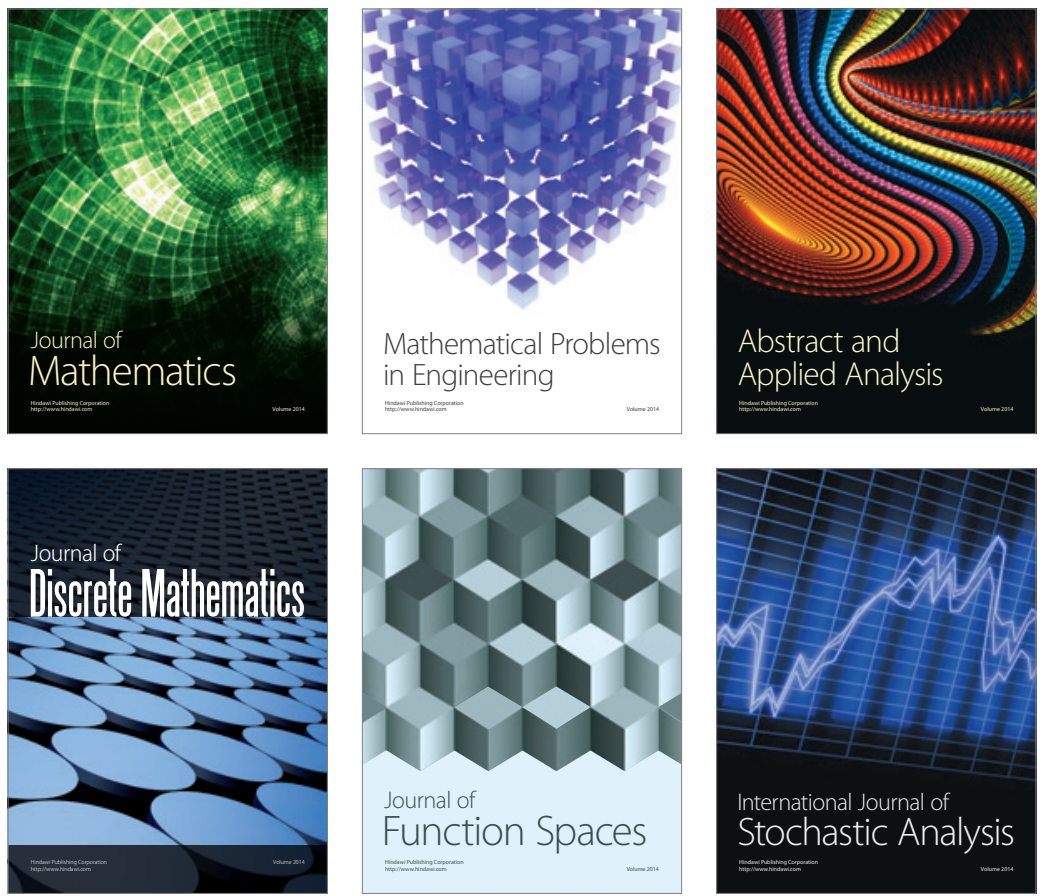

Journal of

Function Spaces

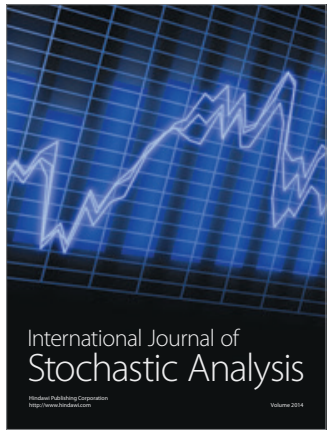

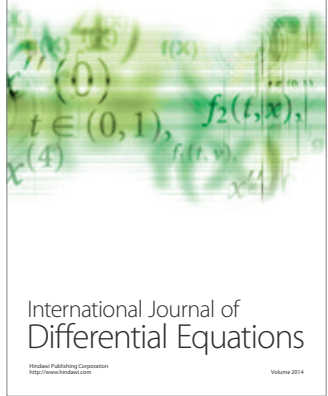
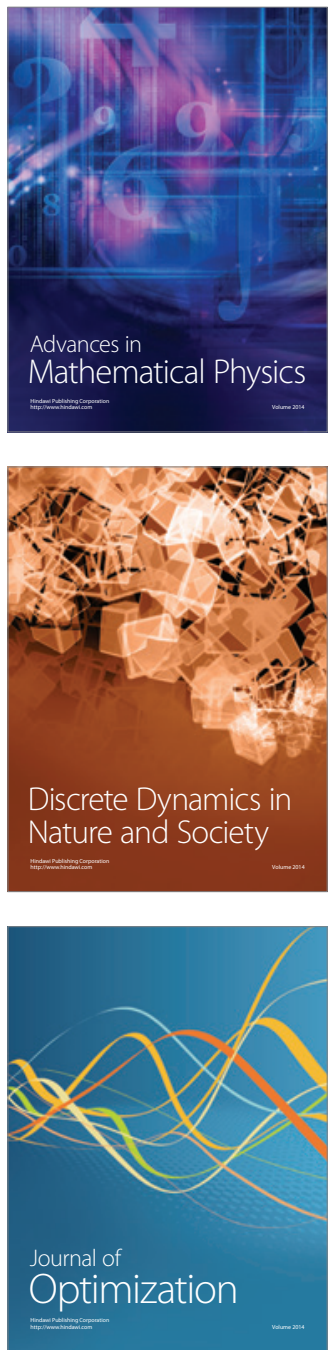